\title{
Production et efficience technique des riziculteurs de Guinée
}

Une estimation paramétrique stochastique

Production and technical efficiency for rice farmers in guinean rural areas with parametric and stochastic estimation

\section{Charlotte Fontan}

\section{OpenEdition}

\section{Journals}

Édition électronique

URL : http://journals.openedition.org/economierurale/342

DOI : 10.4000/economierurale.342

ISSN : 2105-2581

Éditeur

Société Française d'Économie Rurale (SFER)

Édition imprimée

Date de publication : 30 décembre 2008

Pagination : 19-35

ISSN : 0013-0559

\section{Référence électronique}

Charlotte Fontan, «Production et efficience technique des riziculteurs de Guinée », Économie rurale [En ligne], 308 | Novembre-décembre 2008, mis en ligne le 01 novembre 2010, consulté le 10 décembre 2020. URL : http://journals.openedition.org/economierurale/342 ; DOI : https://doi.org/10.4000/ economierurale.342 


\section{Production et efficience technique des riziculteurs de Guinée Une estimation paramétrique stochastique}

Charlotte FONTAN • Groupe de recherche en économie théorique et appliquée (GREThA), Université Montesquieu, Bordeaux

A vec la mise en œuvre des plans d'ajustement structurel dans les pays en développement (PED), les institutions internationales ont soutenu le rôle de la libéralisation agricole notamment pour assurer la sécurité alimentaire, en appuyant les cultures d'exportation qui permettent alors de rembourser la dette de ces pays et de financer les importations alimentaires. Cependant, avec la hausse des prix des denrées alimentaires qui sévit plus durement depuis 2007 et qui provoque des émeutes de la faim dans de nombreux pays, la Banque mondiale et le Fonds monétaire international (FMI) mettent enfin l'accent de façon plus soutenue sur l'agriculture et les filières vivrières.

L'appui à une culture vivrière peut être un moyen efficace de lutter contre l'insécurité alimentaire d'un pays et d'améliorer la situation de l'ensemble des opérateurs d'une filière. La Guinée est un exemple particulièrement intéressant car ce pays dispose de ressources naturelles formidables, mais ne parvient pas à satisfaire ses besoins alimentaires. Il faut partir du constat qu'en Guinée, une très forte majorité d'exploitants agricoles $(75 \%)$, et les plus pauvres d'entre eux, cultivent du riz dans tout le pays, essentiellement pour se nourrir. L'intérêt au départ de produire du riz est très fort dans ce pays car la demande ne cesse d'augmenter. La Guinée a depuis quelques années, surtout avec la libéralisation, recours de façon massive aux importations pour contribuer à la réduction du déficit alimentaire. Certes, la production n'a pas pu suivre la croissance démographique, mais un autre facteur est intervenu : la hausse sensible du niveau de consommation de riz individuel. Plusieurs facteurs justifient un soutien du riz local :

- l'importance du riz en Guinée du point de vue de son apport sur le plan nutritif et donc de sa participation à la garantie de la sécurité alimentaire du pays ;

- le poids de la filière rizicole en termes de surface cultivée, de production et surtout de valeur dégagée par rapport à l'ensemble des autres cultures;

- le rôle que joue cette filière toujours en termes de création de valeur à travers la répartition des revenus entre les différents acteurs, essentiellement issus du milieu rural, concernés en premier lieu par la pauvreté.

Un effort particulier sera nécessaire pour exploiter le formidable potentiel que possède cette filière vivrière. De façon schématique, quatre types de systèmes de production se distinguent en Guinée :

1. Le système de riz pluvial de coteaux (plateau, butte, versants et montagnes) ; 2 . Le système de bas-fond ; 3. Celui de plaine ; 4. Celui de mangrove.

La riziculture pluviale de coteaux reste largement prédominante (65\% des surfaces de riz cultivé). De plus, au niveau de l'approvisionnement des champs en eau, on distingue d'une part le régime pluvial qui concerne la très grande majorité des exploitations, dont l'alimentation dépend totalement des pluies et, d'autre part, le système irrigué ou inondé qui exige des aménagements coûteux, l'eau étant maîtrisée.

Nous nous focaliserons donc sur la culture du riz, aliment indispensable pour les Guinéens, en identifiant des leviers d'amélioration possibles. Cet article déga- 
gera des éléments sur la façon de produire plus de riz, au bénéfice des ménages ruraux, en jouant d'abord sur leur sécurité alimentaire et en générant ensuite davantage de revenus. De plus, nous allons, à travers notre analyse, étudier les moyens susceptibles d'améliorer l'efficience technique des agriculteurs. Le modèle utilisé pour mettre en exergue les outils sur lesquels il faudra agir pour relancer la production et accroître l'efficience technique des riziculteurs est celui de frontière de production stochastique à effets de l'efficience incorporés. L'objectif de ce travail consiste à montrer que des leviers d'amélioration existent pour la filière rizicole. Dans un premier temps, nous développerons le cadre théorique du modèle ainsi que la démarche associée, puis nous mettrons en évidence les variables intervenant dans cette étude. Ensuite, nous appliquerons le modèle proposé par Battese et Coelli en 1995 dans le cadre de la production rizicole guinéenne, ce qui nous permettra de définir des pistes de recommandations de politiques agricoles.

\section{Cadre théorique et présentation des variables}

Il apparaît nécessaire d'introduire le cadre théorique et méthodologique du modèle de frontière de production stochastique et les différents concepts s'y rattachant. Par la suite, nous détaillerons les variables qui seront incluses dans cette application.

\section{Aspects conceptuels, méthodologiques et spécification du modèle}

Cette étude s'articulera selon deux parties : il sera important de définir dans une première section les différents concepts attachés au modèle de frontière de production stochastique puis de mettre en évidence sa démarche ; dans une seconde section, nous en préciserons le cadre méthodologique.

\section{Concepts généraux}

Une fonction de production peut être représentée avec une isoquante qui exprime la frontière de l'ensemble de production, c'està-dire l'output maximum possible à partir d'un niveau donné d'inputs. Efficacité et efficience n'ont pas exactement la même signification, même si ces deux termes sont très proches. Ainsi, l'efficacité fait référence au degré de réalisation d'un objectif souhaité. Quant au mot efficience, il désigne le rapport entre ce qui est réalisé et les moyens mis en œuvre pour y arriver.

Le graphique 1 nous présente le lien entre l'efficience et l'efficacité dans le cadre de notre étude. La finalité première d'appui à la filière rizicole guinéenne est de garantir la sécurité alimentaire du pays au niveau de

\section{Graphique 1. Lien entre efficacité et efficience dans le cadre de notre étude}

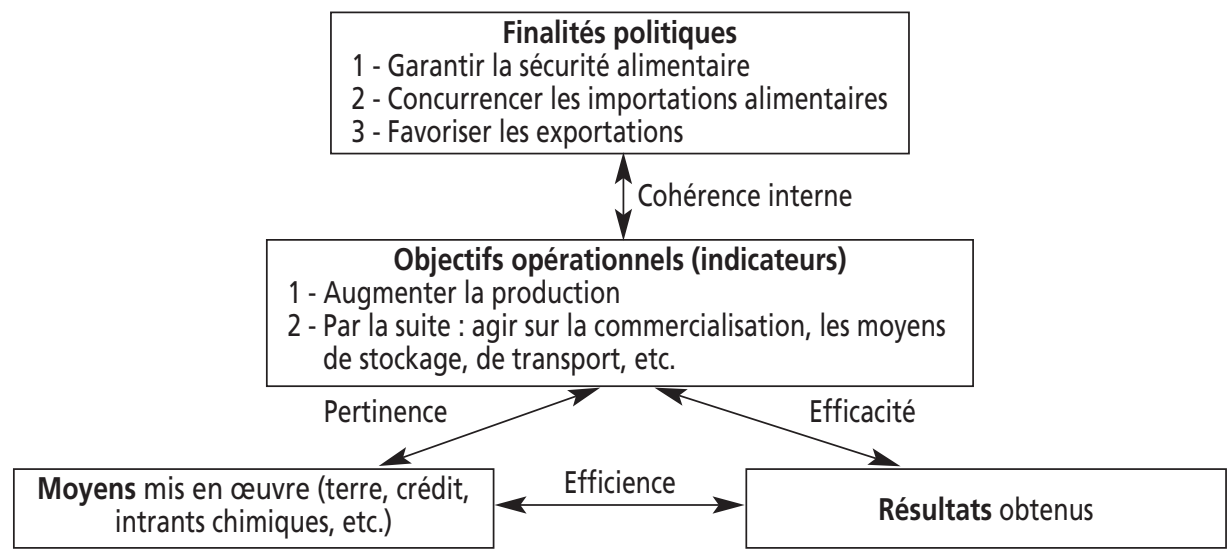

Source : conception de l'auteur, inspirée par Baumstark et al. (2005 - cf. page 64) 
l'alimentation en riz. On privilégiera plutôt la notion d'efficience dans notre étude qui concerne la liaison entre les moyens utilisés (terre, crédit) et le résultat obtenu en terme de production.

Farrell, en 1957, suite aux travaux de Debreu (1951) et Koopmans (1951), a introduit la notion d'efficience technique en proposant une frontière mesurant la performance des entreprises les unes par rapport aux autres. Le concept d'inefficience $\mathrm{X}$ fait suite aux travaux de Farrell avec l'analyse de Leibenstein qui a insisté en 1966, comme Simon (1947), sur l'idée d'une rationalité limitée des agents. Les firmes peuvent se situer structurellement à l'intérieur de leur domaine de possibilité de production $^{1}$. Une entreprise est dite techniquement efficiente si elle se situe sur la frontière des possibilités de production, ce qui signifie qu'en partant d'une quantité de facteurs précise, elle obtient le meilleur niveau d'output réalisable.

\section{Aspects méthodologiques et spécification} du modèle

Pour mesurer l'inefficience technique, on retient l'approche non paramétrique ou paramétrique et la distinction repose sur la forme de la frontière. Si l'on estime que la frontière peut être représentée par une fonction comportant des paramètres explicites, l'approche adoptée sera qualifiée de paramétrique.

Il apparaît que des deux approches (non paramétrique et paramétrique), aucune ne pourra de façon systématique être préférée à l'autre. Selon Coelli et al. (1998), les frontières de type stochastique semblent néanmoins plus appropriées que la méthode non paramétrique dans le domaine agricole, en

1. Cf. Farrell (1957) et Leibenstein (1966).

2. Certains auteurs tentent de rapprocher l'approche stochastique avec celle de la méthode non paramétrique. Cependant, si le modèle a été jusqu'à présent défini au niveau théorique (voir par exemple Cazals et al., 2002 ou Murillo-Zamorano, 2004), nous n'avons pas trouvé d'études empiriques et de logiciels permettant d'utiliser ce modèle non paramétrique et stochastique. particulier pour les $\mathrm{PED}$, où les données sont fortement influencées par des variations aléatoires (comme le climat) ${ }^{2}$.

Le modèle stochastique a été introduit de façon indépendante par Aigner et al. (1977) et Meeusen et Van Den Broeck (1977). Nous appliquerons alors le modèle proposé par Battese et Coelli (1995) qui est une extension de celui développé par les auteurs précédemment cités.

De façon générale, la fonction de production est représentée ainsi :

$$
Y_{i}=f\left(X_{i}, \beta\right) \exp \left(V_{i}-U_{i}\right)
$$

$-Y_{i}$ représente l'output : il s'agira ici de la production de riz de la ième exploitation (Kg obtenu par exploitation) par récolte ; - $X_{i}$ est un vecteur (i*1) des inputs de production associés à la ième exploitation ;

- $\beta$ est un vecteur de paramètres inconnus à estimer ;

- Les $V_{i}$ sont les erreurs aléatoires de distribution $\mathrm{N}\left(0, \sigma_{\mathrm{v}}^{2}\right)$;

- Les $U_{i}$, indépendants des erreurs aléatoires, suivent une loi de distribution tronquée à zéro, de moyenne $m_{i}=z_{i} \delta$ et de variance $\sigma_{u}^{2}$. Les $U_{i}$ sont spécifiés par la fonction suivante : $U_{i}=z_{i} \delta+W_{i}$ avec les $z_{i}$ qui représentent des variables explicatives associées à l'inefficience technique des unités de production, $\delta$ est un vecteur de paramètres inconnus et $W_{i}$ est un terme résiduel.

L'efficience technique s'exprime de la façon suivante :

$$
\begin{aligned}
E T_{i} & =\frac{Y_{i} \text { réalisé }}{Y_{i} \max }=\frac{f\left(X_{i}, \beta\right) \exp \left(V_{i}-U_{i}\right)}{f\left(X_{i}, \beta\right) \exp \left(V_{i}\right)}=\exp \left(-U_{i}\right) \\
& =\exp \left(-\mathrm{z}_{i} \delta-W_{i}\right)
\end{aligned}
$$

La fonction de vraisemblance est exprimée en termes de la variance totale de l'erreur composée $\sigma_{s}^{2}=\sigma_{v}^{2}+\sigma_{u}^{2}$, et de la part de la variance de $U$ dans la variance totale, $\gamma=\sigma_{u}^{2} / \sigma_{s}^{2}$ avec $0<\gamma<1 . \gamma$ représente alors la part relative de la variance expliquée par l'inefficience technique. Dans le modèle retenu, l'écart par rapport à la production 
maximale possible peut être dû, avec l'approche stochastique, à l'inefficience des agriculteurs ou aux facteurs aléatoires qui interviennent pendant le processus de production. Plus la valeur de $\gamma$ est proche de 1 , plus cet écart est surtout attribué à l'inefficience des acteurs, et donc plus les effets aléatoires sont réduits (le modèle serait alors déterministe). Cet indicateur aura un rôle primordial pour justifier la cohérence statistique du modèle.

Les paramètres seront estimés par le programme FRONTIER 4.1 développé par Coelli (1996). Pour la forme de la fonction de production, nous évaluerons dans notre analyse une fonction Cobb-Douglas mais aussi une fonction de type Translog. Cette dernière est plus flexible et permet de calculer des élasticités de substitution, alors que celles-ci sont unitaires dans une fonction Cobb-Douglas (Christensen et al., 1971). Nous décidons donc de prendre en compte deux formes et de comparer les technologies de type Cobb-Douglas et Translog. Un test d'hypothèses permettra d'étudier la forme fonctionnelle la plus appropriée pour notre analyse.

\section{Choix des variables}

\section{Précédents empiriques}

Nous avons introduit, dans l'annexe 1, les résultats d'études réalisées pour mesurer la production et l'efficience technique de riziculteurs dans différents pays à partir de modèles paramétriques stochastiques. Les facteurs étudiés pour la fonction de production et ceux qui mesurent l'efficience ont été séparés. À chaque fois, le coefficient de la superficie est celui qui est le plus élevé pour la production. La superficie a ainsi, pour chaque analyse, un effet significatif et positif sur le volume cultivé, et un coefficient plus fort que les autres facteurs étudiés.

Il faut noter que nous n'avons trouvé que très peu d'études, pour des pays africains, basées sur ce type de modèle et sur la riziculture parmi les recherches que nous avons réalisées ; ce qui renforce l'intérêt de notre analyse. Néanmoins, d'autres travaux sur l'efficience des producteurs de riz, des producteurs de cultures vivrières ou de coton ont été menés en Afrique (voir en particulier Audibert, 1997a ; Audibert et al., 1999).

\section{Variables retenuеs}

Les données mobilisées pour l'analyse proviennent du Recensement national de l'agriculture (RNA). Le RNA a été conduit en Guinée, sur la campagne 2000-2001, dans le cadre du programme de recensement mondial de l'agriculture 2000 préconisé par la FAO. Un travail préalable a dû être effectué avant d'étudier les données recueillies, puisque l'enquête ne portait pas uniquement sur la filière du riz. Nous obtenons alors 828 exploitations. Des variables, qui auraient pu être intéressantes, ne pourront intervenir dans cette analyse, faute de données. Nous verrons, par exemple, qu'il n'y a aucune variable permettant de savoir si le chef de l'exploitation appartient à une organisation paysanne, si les exploitations bénéficient de projets de leurs villages (sur l'éducation, sur différents types d'infrastructures).

Parmi les variables qui seront utilisées, il faut d'abord retenir la variable qui représente l'output. $Y$ représentera la production totale de riz obtenue sur l'ensemble de l'exploitation au cours d'une récolte. Cette variable est exprimée par le poids (en kilogrammes) de riz obtenu sur chaque exploitation.

Au niveau des inputs (les $X_{i}$ de l'équation 1), pour une exploitation agricole, nous décidons de distinguer uniquement les facteurs de production de base : la terre, le capital et le travail. Pour les deux derniers inputs, il est apparu nécessaire d'opérer une distinction, ne pouvant pas désigner le capital et le travail comme deux facteurs parfaitement homogènes. Sont alors considérées deux formes différentes pour le capital (capital rudimentaire et moderne) et pour le travail (travail permanent et 
temporaire). Nous avons donc fait le choix de sélectionner :

- le capital rudimentaire $(\mathrm{K})$ détenu au sein de l'exploitation ${ }^{3}$;

- le nombre de personnes intervenant régulièrement sur l'exploitation $(\mathrm{L}$ : main-d'œuvre familiale et main-d'œuvre extérieure employée de façon permanente c'est-à-dire plus de six mois) ;

- le nombre de jours de travailleurs temporaires utilisés, chaque exploitation ayant recours à une main-d'œuvre extérieure pour une durée variant d'un jour à six mois au maximum (MoT) ;

- la superficie de riz cultivée (SuperRiz) ;

- le fait que l'exploitant agricole utilise ou pas du capital moderne (CapMod : tracteur, herse attelée, motorisée, charrue).

Pour les facteurs susceptibles d'agir sur l'efficience (les $U_{i}=z_{i} \delta+W_{i}$ ), par rapport aux données disponibles, il sera intéressant de tester :

- le régime hydrique (REGHYD) sur lequel le riz est cultivé, afin de déterminer s'il vaut mieux une riziculture irriguée ou pluviale. Il faut tenir compte évidemment du système de culture et nous avons vu en introduction que le riz peut être cultivé sur quatre types de systèmes différents. Il apparait toutefois que, plus que le type de relief, le régime hydrique est déterminant puisque les différents types de système peuvent être caractérisés par un régime pluvial ou irrigué ; - le fait que l'exploitation se situe dans une région naturelle favorable (RÉGION), sur le plan des conditions naturelles et de leur capacité de production. Deux régions se distinguent favorablement des deux autres au niveau de leur potentiel : la Guinée maritime et la Guinée forestière ;

- une variable rendant compte de l'effet de l'utilisation d'au moins un type d'intrants

3. Nous reprenons entre parenthèses, pour tous les inputs et les variables agissant sur l'efficience technique, les abréviations qui seront utilisées dans les équations 3 et 5 . chimiques (INTRANTS parmi fumure organique, engrais azoté, phosphaté, potassé, triple ou traitement phytosanitaire) sur l'efficience technique ;

- des variables caractéristiques sur le chef de ménage au niveau de son sexe (SEXE), son âge (AGE) et de son niveau d'alphabétisation (ALPHA) ;

- le fait de ne cultiver que du riz sur l'ensemble de l'exploitation (MONOC), et ainsi de voir si une spécialisation est plus favorable à l'efficience technique que la diversification des cultures, censée limiter les risques liés à l'agriculture ;

- l'importance des activités non agricoles (ACTNONAGR), là encore pour tester le rôle de la diversification (des activités cette fois-ci) ;

- le fait de recourir à un crédit (officiel ou non : CREDIT) ;

- le nombre total de champs que l'on peut délimiter sur l'exploitation (NbreCHAMPS) ; - une variable tenant compte du rôle de la propriété des terres (PROP) pour définir s'il est bénéfique, pour l'efficience technique des riziculteurs, d'être propriétaire d'une exploitation.

L'annexe 2 reprend la description et les statistiques descriptives des variables introduites dans notre modèle.

\section{Estimation d'une fonction de production paramétrique et stochastique}

Le modèle ayant été décrit, tout comme les variables du modèle stochastique incorporant les effets de l'efficience, il est maintenant temps de l'appliquer. Ensuite, il s'agira d'analyser les résultats obtenus, ce qui donnera des orientations pour les politiques agricoles à mettre en œuvre.

\section{Application du modèle paramétrique et stochastique}

D'abord, la frontière de production sous une technologie de type Cobb-Douglas s'écrit ainsi : 
$\ln Y_{i}=\beta_{0}+\beta_{1} \ln K_{i}+\beta_{2} \ln L_{i}+\beta_{3} \ln M o T_{i}+\beta_{4}$ In SuperRiz $_{i}+\beta_{5}$ CapMod $_{i}-U_{i}+V_{i}{ }^{4}$

Ensuite, une fonction de production de type Translog est définie par la forme suivante :

$\ln Y_{i}=\beta_{0}+\sum_{j=1}^{5} \beta_{j} \ln X_{j i}+\frac{1}{2} \sum_{j=1}^{5} \sum_{k=1}^{5} \beta_{j k} \ln X_{j i}$

$$
\ln X_{k i}-U_{i}+V_{i}
$$

Pour les deux types de fonction, les effets de l'inefficience technique sont définis par l'équation (5) :

$m_{i}=\delta_{0}+\delta_{1} R_{E G H Y D}+\delta_{2}$ REGION $_{i}+\delta_{3}$ INTRANTS $_{i}$ $+\delta_{4} S E X E_{i}+\delta_{5} A G E_{i}+\delta_{6} A L P H A_{i}+\delta_{7} M O N O C_{i}+$ $\delta_{8}$ ACTNONAGR $_{i}+\delta_{9}$ CREDIT $_{i}+\delta_{10}$ NbreCHAMPS $_{i}$ $+\delta_{11}$ PROP $_{i}$

avec $m_{i}$ qui définit la moyenne de l'efficience technique $U_{i}$ (pour i allant de 1 à 828 exploitations).

\section{Estimation des fonctions Cobb-Douglas et Translog}

Il fallait effectuer un choix arbitraire quant à la distribution de $U$ puisque celle-ci n'en a pas a priori, ce qui est d'ailleurs une des critiques fréquentes du modèle stochastique.

Nous supposons que cette variable suit une loi normale tronquée, distribution considérée comme étant plus réaliste par beaucoup d'auteurs (consulter par exemple Murillo-Zamorano, 2004).

Les résultats du modèle, dans le cas où on retient une fonction Cobb-Douglas, sont donnés dans l'annexe 3. L'avantage d'une telle fonction est de pouvoir donner directement les élasticités à partir des valeurs des coefficients. Il convient également de présenter les résultats obtenus dans le cas où la fonction de production est sous une forme Translog, ce que nous avons réalisé dans le tableau 1. Avant d'interpréter l'ensemble

4. À noter que nous ne prendrons pas le logarithme de la variable «Utilisation de capital moderne » car celle-ci est dichotomique. des coefficients, nous discuterons de la cohérence statistique du modèle et nous effectuerons différents tests d'hypothèses dont un qui nous permettra de retenir la forme Translog comme étant plus adaptée que la forme Cobb-Douglas.

\section{Cohérence statistique du modèle}

La valeur de gamma est importante afin de justifier l'intérêt de notre étude. Dans notre cas, il faut d'abord prendre en compte que $\gamma$ est fortement significatif (à $1 \%$ ) et que ce paramètre atteint une valeur de 0,54 et 0,59 , selon la forme de la fonction de production. Cela signifie que l'inefficience technique des riziculteurs guinéens joue un rôle important pour expliquer la distance par rapport à la frontière de production, mais que le rôle des facteurs aléatoires est tout aussi important, ce qui correspond à un résultat cohérent avec les spécificités du secteur agricole. Cette étape nous permet de justifier l'intérêt d'avoir considéré une frontière stochastique plutôt que déterministe puisque cette dernière néglige les effets des facteurs aléatoires.

Il semble aussi important d'étudier si une des deux formes de la fonction de production est plus adaptée que l'autre. Pour cela, à l'aide des LR (Likelihood ratio) donnés par le logiciel utilisé, nous testons l'hypothèse suivante $\mathrm{H}_{\mathrm{o}}: \beta_{j k}=0$. Ainsi, si $\mathrm{H}_{\mathrm{o}}$ est acceptée et que les coefficients $\beta_{j k}$ sont nuls, la fonction sous forme Cobb-Douglas est la plus adaptée. Le LR test entre l'hypothèse nulle $\mathrm{H}_{\mathrm{o}}$ et l'hypothèse alternative $\mathrm{H}_{1}$ est donné directement par l'application du modèle et repose sur la statistique suivante :

$\mathrm{LR}=-2\left\{\ln \left[\mathrm{L}\left(\mathrm{H}_{\mathrm{o}}\right) / \mathrm{L}\left(\mathrm{H}_{1}\right)\right]\right\}=-2\left\{\ln \left[\mathrm{L}\left(\mathrm{H}_{\mathrm{o}}\right)\right]\right.$ $\left.-\ln \left[\mathrm{L}\left(\mathrm{H}_{1}\right)\right]\right\}$, où $\mathrm{L}\left(\mathrm{H}_{\mathrm{o}}\right)$ et $\mathrm{L}\left(\mathrm{H}_{1}\right)$ correspondent aux fonctions de vraisemblance pour les hypothèses $\mathrm{H}_{0}$ et $\mathrm{H}_{1}$ et représentent donc les valeurs des rapports de vraisemblance respectivement pour les fonctions CobbDouglas et Translog. Cette statistique suit une loi de Khi-deux mixte dont le nombre de degrés de liberté est équivalent au nombre 
RECHERCHES

Charlotte FONTAN

Tableau 1. Résultats de l'analyse en frontière de production stochastique dans le cas d'une fonction Translog

\begin{tabular}{|c|c|c|c|}
\hline Variables $^{1}$ & & Coefficients $^{2}$ & $t$ de Student \\
\hline \multicolumn{4}{|l|}{ Coefficients agissant sur la fonction de production } \\
\hline Constante & $\beta_{0}$ & $8,75 * * *$ & 27,10 \\
\hline Capital rudimentaire détenu $(K)$ & $\beta_{1}$ & $-0,63 * *$ & $-2,40$ \\
\hline Nombre d'actifs agricoles permanents $(\mathrm{L})$ & $\beta_{2}$ & $0,31 *$ & 1,67 \\
\hline Nombre de jours d'actifs temporaires utilisés (MoT) & $\beta_{3}$ & $-0,02$ & $-0,31$ \\
\hline Superficie de riz cultivée (en Ha) (SuperRiz) & $\beta_{4}$ & $0,85 * * *$ & 5,27 \\
\hline Emploi de capital moderne (CapMOD) & $\beta_{5}$ & 0,23 & 0,78 \\
\hline $\mathrm{K} * \mathrm{~K}$ & $\beta_{6}$ & $0,48 * * *$ & 3,04 \\
\hline$L * L$ & $\beta_{7}$ & $-0,16^{*}$ & $-1,70$ \\
\hline MoT*MoT & $\beta_{8}$ & 0,006 & 0,41 \\
\hline SuperRiz*SuperRiz & $\beta_{9}$ & $-0,30$ & $-0,33$ \\
\hline CapMOD*CapMOD & $\beta_{10}$ & $0,002 *$ & 1,86 \\
\hline $\mathrm{K} * \mathrm{~L}$ & $\beta_{11}$ & $-0,10$ & $-0,51$ \\
\hline $\mathrm{K} * \mathrm{MoT}$ & $\beta_{12}$ & $0,14 * *$ & 2,13 \\
\hline K*SuperRiz & $\beta_{13}$ & $-0,12$ & $-0,66$ \\
\hline K*CapMOD & $\beta_{14}$ & $-0,49$ & $-1,52$ \\
\hline L*MoT & $\beta_{15}$ & $-0,04$ & $-1,05$ \\
\hline L*SuperRiz & $\beta_{16}$ & 0,10 & 0,92 \\
\hline L*CapMOD & $\beta_{17}$ & $-0,02$ & $-0,10$ \\
\hline MoT*SuperRiz & $\beta_{18}$ & 0,04 & 1,06 \\
\hline MoT*CapMOD & $\beta_{19}$ & $-0,11 *$ & $-1,88$ \\
\hline SuperRiz*CapMOD & $\beta_{20}$ & $0,27 * *$ & 1,96 \\
\hline \multicolumn{4}{|l|}{ Coefficients agissant sur l'inefficience technique 3} \\
\hline Constante & $\delta_{0}$ & $1,47 * * *$ & 9,42 \\
\hline Régime hydrique & $\delta_{1}$ & $-1,23 * * *$ & $-2,95$ \\
\hline Région naturelle favorable & $\delta_{2}$ & 0,12 & 1,13 \\
\hline Utilisation d'au moins un intrant chimique & $\delta_{3}$ & 0,03 & 0,61 \\
\hline Chef de ménage masculin & $\delta_{4}$ & 0,03 & 0,31 \\
\hline Age du chef de ménage & $\delta_{5}$ & $-0,003 * * *$ & $-2,37$ \\
\hline Niveau d'alphabétisation & $\delta_{6}$ & $-0,05$ & $-0,12$ \\
\hline Monoculture pratiquée & $\delta_{7}$ & $-0,10 * * *$ & $-2,60$ \\
\hline Actif de l'exploitation dans activités non agricoles & $\delta_{8}$ & 0,07 & 0,24 \\
\hline Recours à un crédit & $\delta_{9}$ & $-0,04^{*}$ & $-1,81$ \\
\hline Nombre total de champs & $\delta_{10}$ & $-0,03 * *$ & $-1,97$ \\
\hline Propriété de champs & $\delta_{11}$ & $-0,17^{*}$ & $-1,69$ \\
\hline Gamma ${ }^{4}$ & $\gamma_{4}$ & $0,59 * * *$ & 3,34 \\
\hline
\end{tabular}

Notes (1) Variable exogène : la quantité de riz cultivée ; (2) *Significatif à $10 \%$; **Significatif à $5 \%$; ** Significatif à $1 \%$. Il faut bien tenir compte du fait que les coefficients obtenus au départ pour les différents facteurs de production ne donnent pas les valeurs des élasticités mais celles-ci seront obtenues à partir d'un calcul que nous détaillerons par la suite. Ils ne peuvent donc pas être interprétés tels quels ; (3) On mesure bien ici la relation entre les différentes variables retenues et l'inefficience technique ; (4) Gamma représente la part de la variance de U, qui désigne les facteurs de l'inefficience technique, dans la variance totale. Ce coefficient permet de discuter de la cohérence statistique du modèle. 
Tableau 2. Tests d'hypothèses pour la fonction Translog

\begin{tabular}{l|c|c|c}
\hline Hypothèse nulle & LR & $\begin{array}{l}\text { Statistique du Khi deux } \\
\text { Mixte (à } 5 \% \text { ) }\end{array}$ & Décision \\
\hline $\mathrm{T}_{1}: \mathrm{H}_{0}: \beta_{\mathrm{jk}}=0$ & 32,14 & 24,38 & Ho rejetée \\
$\mathrm{T}_{2}: \mathrm{H}_{0}: \gamma=0$ & 45,11 & 2,71 & Ho rejetée \\
$\mathrm{T}_{3}: \mathrm{H}_{0}: \delta_{0}=\delta_{1}=\ldots=\delta_{11}=0$ & 57,93 & 17,67 & Ho rejetée \\
\hline
\end{tabular}

Source : calculs de l'auteur, d'après les calculs effectués sur FRONTIER 4.1 à partir du RNA de la FAO réalisé en 2000-2001

de restrictions imposées ${ }^{5}$. $\mathrm{H}_{0}$ sera alors rejetée si LR $>\chi^{2}(n, \alpha)^{6}$.

L'hypothèse $\mathrm{H}_{\mathrm{o}}$ est ici rejetée, comme nous le voyons dans le tableau 2 (test 1 $\mathrm{T}_{1}$ ), ce qui signifie qu'une forme Translog est plus adaptée pour notre étude. Pour la suite de cette analyse et l'interprétation des résultats, nous parlerons donc surtout des coefficients obtenus par l'application du modèle dans la fonction Translog ${ }^{7}$. Deux autres tests vont être effectués uniquement pour la fonction sous forme Translog. Le premier (marqué T2 au tableau 2) compare l'hypothèse $\mathrm{H}_{0}: \gamma=0$ avec l'hypothèse alternative $\mathrm{H}_{1}: \gamma>0$. Il s'agit à nouveau de voir si la frontière de production stochastique est appropriée dans notre cas. La valeur critique, donnée directement par le logiciel avec le LR, suit là encore une distribution Khi-deux mixte. Nous testons enfin une autre hypothèse (test $\mathrm{T}_{3}$ ), toujours pour la forme Translog, $\mathrm{H}_{\mathrm{o}}: \delta_{0}=\delta_{1}=\ldots=\delta_{11}=0$ afin de savoir s'il est légitime d'étudier les effets liés à l'inefficience technique ${ }^{8}$. D'après les résultats du tableau 2 , nous voyons que les trois hypothèses testées sont rejetées à $5 \%$. Les valeurs du LR correspondent à

5. Table à lire dans Kodde et Palm (1986, p. 1246). 6. Le nombre de restrictions est celui qui différencie les deux équations testées. $n$ désigne le nombre de restrictions (ou degrés de liberté) et $\alpha$ le seuil de tolérance.

7. Les résultats sont de toute façon similaires entre les deux types de fonction pour toutes les variables de l'efficience.

8. Pour trouver la valeur du LR, il faut déterminer celle de $\mathrm{L}\left(\mathrm{H}_{\mathrm{o}}\right)$ en appliquant le modèle FRONTIER dans le cas où aucun facteur d'inefficience n'intervient donc juste avec les facteurs de production. celles des résultats de la fonction Translog mais les calculs que nous avons effectués par ailleurs pour la forme Cobb-Douglas ont donné les mêmes conclusions pour les deux derniers tests.

$\mathrm{Au}$ regard des différents tests, nous pouvons conclure que la forme la plus adaptée pour la fonction de production, entre la fonction Cobb-Douglas et Translog, est bien cette dernière et que la forme stochastique est plus appropriée. Nous avons démontré la validité du modèle étudié ici et nous pouvons alors considérer avec intérêt les résultats de notre étude.

\section{Analyse des effets du modèle de la fonction sous forme Translog}

L'analyse va être effectuée en deux temps puisqu'il s'agira de voir d'abord les résultats des facteurs de production puis ceux de l'inefficience technique.

\section{Coefficients de la fonction de production}

À partir d'une fonction Translog, la lecture des coefficients n'est pas directe comme la fonction Cobb-Douglas et les élasticités des facteurs de production sont calculées ainsi

$$
\varepsilon_{j}=\frac{\partial \ln (y)}{\partial \ln \left(x_{j}\right)}=\beta_{j}+\sum_{j} \beta_{j k} \ln \left(x_{k}\right)
$$

On ne peut donc pas lire directement les coefficients du tableau 1 pour les facteurs de production. Le tableau 3 donne les valeurs moyennes, minimales puis maximales des élasticités des cinq facteurs de production. Dans l'interprétation des résultats, nous allons juste considérer les élasticités moyennes.

Parmi les coefficients estimés, le facteur 
Tableau 3. Valeurs des élasticités directes des facteurs de production

\begin{tabular}{c|c|c|c}
\hline Facteurs de production & Moyenne & Minimum & Maximum \\
\hline Capital rudimentaire & 0,93 & $-0,39$ & 1,68 \\
Travail permanent & $-0,26$ & $-0,50$ & 0,16 \\
Travail temporaire & 0,11 & $-0,05$ & 0,20 \\
Superficie & 0,95 & 0,91 & 1,17 \\
Capital moderne & $-0,51$ & $-1,82$ & 0,13 \\
\hline
\end{tabular}

Source : calculs de l'auteur, d'après les calculs effectués à partir du RNA de la FAO réalisé en 2000-2001

travail que l'on pourrait qualifier de régulier, et calculé par le nombre de travailleurs issus de la famille et ceux qui sont employés de façon permanente (plus de six mois pendant l'année), a un effet significatif et négatif. Ce résultat signifie donc que la production rizicole diminuerait, toutes choses égales par ailleurs, avec le nombre de travailleurs permanents. Si la productivité marginale du travail est reconnue pour être décroissante dans le secteur agricole (l'apport d'un travailleur supplémentaire sur la production est de plus en plus faible), notre résultat indique même que le nombre de travailleurs permanents fait baisser la production. On atteint ici un cas extrême et il semble que la réalité se rapprocherait plus d'un effet non significatif du travail permanent sur la production, que nous avons défini à partir de la fonction sous forme Cobb-Douglas. Dans tous les cas, il faut compter aujourd'hui sur d'autres facteurs pour améliorer le niveau de production rizicole. Ce résultat est très important puisqu'il souligne le problème d'affectation du travail ainsi que le phénomène de sous-emploi lié à l'intensification du travail, particulièrement fréquent dans les milieux ruraux africains, surtout pendant les périodes agricoles creuses et parce que quasiment tous les membres de l'exploitation travaillent sur les terres agricoles. Cet effet est néanmoins à relativiser au regard de l'importance, au sein de nombreuses exploitations agricoles africaines, de la pluriactivité pour les membres de celles-ci. La variable retenue représente en effet les travailleurs qui se consacrent aux activités agricoles mais on ne connaît pas exactement leur degré d'implication pour la culture du riz ${ }^{9}$. Des hypothèses sur ce problème de sous-emploi peuvent être émises. Le manque de motivation de certains actifs lié, par exemple, aux difficultés d'enrichissement par la production de riz (problèmes de commercialisation ou de stockage) en serait l'une des raisons. Une autre explication sur un tel comportement serait qu'ils n'auraient pas forcément la récompense de leurs efforts ${ }^{10}$.

De la même façon, l'emploi de capital moderne influence, de façon significative et négative, la production de riz. Un tel résultat pourrait s'expliquer par une mauvaise utilisation ou maîtrise de ce type de capital et donc par un manque de formation. Il faut néanmoins rester prudent avec ces affirmations puisque seulement $15 \%$ des exploitations ont recours à des tracteurs ou à la culture attelée. Le problème peut aussi venir de la substitution à d'autres variables : ainsi, l'usage de tracteur ou de herse motorisée par exemple peut remplacer du temps de travail.

Pour les trois autres facteurs de production testés, tous significatifs, nous pouvons observer que la production dépend plus largement de l'augmentation des surfaces cultivées (coefficient de 0,95) que de l'utilisation du capital rudimentaire $(0,93)$ ou que

9. Cependant, sur l'ensemble des exploitations retenues, la culture rizicole représente plus de $75 \%$ en moyenne de leur superficie totale, ce qui laisse supposer un rôle important joué par cette filière. 10. Par exemple, les derniers enfants d'une famille peuvent être moins incités à produire que leurs aînés, sachant qu'ils n'hériteront sûrement pas de la terre de leurs parents. 
du nombre de jours travaillés par la maind'œuvre extérieure temporaire $(0,11)$ qui, elle, semble productive.

Il faut noter que les travailleurs temporaires jouent un rôle en matière de production. Il apparaît important de recourir à ce type de main-d'œuvre pour combler le manque de productivité des travailleurs permanents ${ }^{11}$. Une différence existe donc entre le travail permanent et le travail temporaire qui intervient uniquement quand les chefs de l'exploitation en ont besoin, qui est donc nécessaire, voire même indispensable au regard des résultats obtenus. Un appui à la filière rizicole, en amont de celle-ci, nécessite surtout une action sur les travailleurs réguliers, mais il ne faudrait pas oublier qu'une meilleure maîtrise du capital moderne sera aussi importante, si on décide d'étendre son utilisation. De plus, le potentiel naturel guinéen n'est certes pas encore exploité en totalité au niveau des terres agricoles mais l'extension des surfaces cultivées aura à un moment des limites, ce qui accentuera le rôle que devront jouer les autres facteurs de production.

Une fonction de type Translog nous permet aussi de calculer des élasticités de substitution entre les facteurs de production. Nous avons évalué les élasticités de substitution d'Allen et de Morishima et elles sont, pour la plupart, très proches de la valeur nulle, ce qui met en évidence une relation inélastique entre les facteurs concernés ${ }^{12}$.

\section{Coefficients agissant sur l'inefficience}

Dans un premier temps, cinq variables sont caractérisées par un coefficient non significatif. Ainsi, le fait de cultiver dans une région plus favorisée, l'utilisation des intrants chimiques, le sexe du chef de

11. Résultat similaire à celui trouvé par Ogundele et Okoruwa (2004) introduit dans le tableau de l'annexe 1.

12. Pour des détails sur le calcul des élasticités de substitution, consulter Allen (1938), Blackorby and Russell (1989) ou Morishima (1967). ménage, son niveau d'alphabétisation et le fait qu'un membre de l'exploitation pratique des activités non agricoles ne sont pas des causes de l'efficience technique. Pour le sexe et les intrants chimiques, les résultats doivent être observés avec prudence au regard de leur distribution ${ }^{13}$. De tels résultats peuvent paraître contraires à certaines idées reçues. En effet, de nombreuses politiques et institutions préconisent l'importance de l'éducation pour améliorer le sort des plus démunis, notamment en zones rurales ainsi que le recours aux intrants chimiques. Néanmoins, les personnes plus éduquées peuvent se désintéresser de l'exploitation agricole en se consacrant à d'autres activités, plus rémunératrices ou plus intellectuelles. Un effet «d'allocation » ou « de qualification » peut jouer en effet ici. Des auteurs le définissent ainsi : "l'éducation conduit les agents comparativement éduqués à allouer temps et travail à des activités qui, du point de vue de leurs critères, maximisent davantage leur utilité, quelle qu'elle soit » (Audibert, Mathonnat, Nzeyimana et Henry, 1999).

Même s'il ne faut pas oublier que très peu d'exploitants détiennent des intrants chimiques, on peut conclure que l'utilisation de ceux-ci n'est, à l'heure actuelle, pas adaptée pour cultiver du riz en Guinée ou alors que les Guinéens n'ont pas la capacité et la formation de bien recourir à ce type d'intrants. Là encore, ce résultat pourrait être expliqué par un effet de substitution, ce qui paraît comme étant une raison plus valable ici.

En outre, cultiver dans une région naturelle plus favorisée n' agit pas positivement sur l'efficience technique des riziculteurs. Une plus grande efficience, qui pourrait expliquer de meilleurs résultats en matière de production, ne dépend pas de la situation géographique en Guinée mais est fonction d'autres variables comme l'implication des

13. Cf. annexe 2 . 
acteurs pour mettre l'accent sur la filière rizicole par exemple. Enfin, si un actif permanent du ménage pratique des activités non agricoles, l'effet est non significatif pour l'efficience de l'exploitation. Pourtant, l'importance de diversifier les cultures et les activités est très souvent préconisée afin de faire face aux risques.

Dans un second temps, nous verrons les variables qui favorisent l'efficience technique des riziculteurs. D'abord, un système irrigué a un effet significatif et positif sur l'efficience technique. La riziculture guinéenne, essentiellement pratiquée de façon pluviale, doit donc axer ses efforts sur l'irrigation, techniquement plus difficile et qui demande des aménagements importants. En outre, l'âge du chef d'exploitation est un déterminant de l'efficience : les exploitants âgés sont plus efficients que les exploitants jeunes. L'effet négatif du capital moderne sur la production et la nonsignificativité de l'utilisation d'intrants chimiques sur l'efficience technique peuvent expliquer que l'âge joue ici un rôle positif. Il apparaît que l'on se situe dans un pays où l'agriculture reste très traditionnelle. La production et l'efficience reposent donc pour l'instant plus sur l'âge et l'expérience des actifs agricoles que sur la modernisation des techniques employées.

Ensuite, le fait de ne cultiver que du riz au sein d'une même exploitation, sur l'ensemble des parcelles et des champs de celleci, agit aussi favorablement sur l'efficience technique. Pourtant, beaucoup d'agriculteurs pratiquent la pluriculture au niveau agricole car elle permet de diversifier les risques et de s'en prémunir. D'après nos résultats, il semblerait souhaitable, au moins au départ, de ne favoriser que la culture du riz. $\mathrm{Ce}$ résultat se rapproche de l'impact non significatif de la variable qui mesure le fait pour des membres des exploitations de pratiquer des activités non agricoles. Comme le modèle utilisé nous donne uniquement la relation entre la monoculture et l'efficience, on peut juste conclure que les riziculteurs les plus efficients, au cours d'une période donnée (une récolte), sont ceux qui se spécialisent dans une seule culture et une seule activité, ce qui paraît alors logique. En revanche, on ne peut pas recommander la monoculture de façon systématique car si la pluriactivité agricole ne favorise pas l'efficience, elle permet de diversifier les risques. L'arbitrage devra donc se faire entre une plus grande efficience à un moment donné et une diversification des risques à plus long terme.

Si un riziculteur utilise un crédit pour ses activités, il sera plus efficient, ce qui confirme l'effet bénéfique attendu d'un recours au crédit. Le nombre de champs, qui définit le morcellement d'une exploitation, a un impact positif sur l'efficience technique. On pourrait expliquer cette relation par le fait qu'une dispersion des champs, d'un point de vue géographique, puisse permettre de diversifier les risques. Une même exploitation peut en effet se situer sur des types de reliefs différents et de meilleurs résultats seront observés sur un champ particulier. Enfin, le fait qu'un membre permanent de l'exploitation soit propriétaire d'une parcelle contribue positivement à l'efficience technique. Il faut donc favoriser, par l'imposition de règles foncières spécifiques, l'accès à la propriété. Au niveau de la valeur des coefficients, la pratique de la riziculture irriguée a un effet plus important sur l'efficience technique des riziculteurs guinéens que celui d'être propriétaire, de ne cultiver que du riz, d'avoir recours à un crédit, d'avoir un nombre de champs élevé ou d'être âgé.

Le modèle FRONTIER 4.1 permet également d'évaluer l'efficience technique de chaque exploitation et ainsi l'efficience moyenne des riziculteurs en Guinée ${ }^{14}$. Pour l'ensemble des exploitations, celle-ci s'élève à 0,66 . Plus on s'approche de 1 , plus la distance par rapport à la frontière de production est faible. Pour comparer, Gragasin et

14. Le calcul est effectué directement par le logiciel, en partant de l'équation (2). 
al. (2005), en étudiant l'efficience technique de riziculteurs aux Philippines, ont trouvé un niveau moyen de 0,80. En Inde, d'autres auteurs ont étudié la riziculture et la moyenne de l'efficience technique s'élève alors à 0,86 (Fuwa et al., 2005). De même, en Indonésie, une étude a trouvé un niveau de 0,84 (Daryanto et al., 2002). Ces quelques données montrent, comme nous pouvions l'attendre, que la riziculture asiatique est plus efficiente que celle pratiquée en Guinée. La valeur de l'efficience des exploitations guinéennes confirme qu'il existe des marges de manœuvre pour la riziculture guinéenne, tant qu'elle n'atteint pas la valeur unitaire.

\section{Conclusion}

Cet article cherchait en premier lieu à démontrer que des leviers d'amélioration existent pour la filière rizicole guinéenne et pas à énumérer tous les instruments et méthodes permettant de produire plus de riz en Guinée, qui relèvent du travail des agronomes. Au regard de l'évolution actuelle des cours sur les marchés internationaux, une formidable opportunité s'offre pour les pays qui ont un potentiel agricole inexploité et en particulier pour les cultures rizicoles ouest-africaines.

Si la production et l'efficience technique ne semblent pas encore liées à l'introduction d'intrants plus modernes, sûrement parce que l'agriculture reste encore très traditionnelle en Guinée, un effort sensible doit, d'abord et avant tout, être orienté vers les travailleurs permanents des exploitations rizicoles. Par la suite, il sera bien évidemment important d'engager des actions vers une plus grande modernité des techniques et des moyens utilisés.

Même s'il est évident que les politiques agricoles ne devront pas se focaliser uniquement sur la culture du riz, pour se préserver des risques, et que le développement rural en Guinée sera incontestablement fonction de la détermination de politiques plus larges non seulement sur les autres cultures, mais également sur l'ensemble des activités non agricoles, la filière rizicole dispose d'un potentiel formidable. Il faudra alors faire face à un ensemble de contraintes. La plus importante repose sur le problème de compétitivité entre le riz guinéen et le riz asiatique, celuici étant importé à un prix encore plus faible, malgré la flambée des cours des produits agricoles sur les marchés internationaux. 


\section{RÉFÉRENCES BIBLIOGRAPHIQUES}

Aigner D.-J., Lovell C.-A., Schmidt P. (1977). Formulation and estimation of stochastic frontier production functions models. Amsterdam, North Holland Publication, Journal of Econometrics, $\mathrm{n}^{\circ}$ 6, p. 21-37.

Allen R.-G.-D. (1938). Mathematical analysis for economists. London, MacMillan.

Audibert M. (1997a). La cohésion sociale est-elle un facteur de l'efficience technique des exploitations agricoles en économie de subsistance ? Paris, Presses Universitaires de France, Revue d'Économie du Développement, $\mathrm{n}^{\circ} 3$, p. 69-90.

Audibert M. (1997b). Technical inefficiency effects among paddy farmers at the 'Office du Niger', Mali, West Africa. Springer Netherlands, Journal of Productivity Analysis, vol. 8, n 4, p. 379-394.

Audibert M., Mathonnat J., Nzeyimana I., Henry M.-C. (1999). Rôle du paludisme dans l'efficience technique des producteurs de coton au nord de la Côte-d'Ivoire. Paris, Presses Universitaires de France, Revue d'Économie du Développement, $\mathrm{n}^{\circ} 4$, p. 121-148.

Battese G., Coelli T. (1995). A model for technical inefficiency effects in a stochastic frontier production function for panel data. Physica Verlag, Empirical Economics, vol. 20, $\mathrm{n}^{\circ}$ 2, p. 325-332.

Baumstark L., Menard C., Roy W., Yvrande-Billon D. (2005). Mode de gestion et efficience des opérateurs dans le secteur des transports urbains de personnes. Lyon : LET, Paris : Centre ATOM, 154 p.

Blackorby C., Russell R. (1989). Will the real elasticity of substitution please stand up? (A comparison of the Allen/Uzawa and Morishima Elasticities). Nashville, American Economic Association, The American Economic Review, vol. 79, $n^{\circ} 4$, p. 882-887.
Cazals C., Florens J.-P., Simar L. (2002). Non parametric frontier estimation: a robust approach. Amsterdam, North Holland Publication, Journal of Econometrics, $\mathrm{n}^{\circ} 106$, p. 1-25.

Christensen L.-R., Jorgenson W., Lau L.-J. (1971). Conjugate duality and the transcendental logarithmic production function. Chicago, the University of Chicago, Econometric Society, Econometrica, vol. 39, p. 255-256.

Coelli T., Rao P., Battese G. (1998). An introduction to efficiency and productivity analysis. Massachusetts, Kluwer Academic Publishers, 275 p.

Coelli T. (1996). A guide to frontier, version 4.1: A computer program for stochastic frontier production and cost function estimation. Australie, Draft, Armidale, 33 p. Daryanto H.-K.-S., Battese G.-E., Fleming E.M. (2002). Technical efficiencies of rice farmers using different irrigation systems in different cropping seasons in West Java, Indonesia, School of Economics, University of New England, Armidale, NSW, CEPA Working Papers, $\mathrm{n}^{\circ}$ 1, $27 \mathrm{p}$.

Debreu G. (1951). The coefficient of ressource utilization. Chicago, the University of Chicago, Econometric Society, Econometrica, vol. 19, n 3, p. 273-292.

Farrell M.-J. (1957). The measurement of productive efficiency. Londres, Royal Statistical Society, Cambridge, Journal of the Royal Statistical Society, Series A, $\mathrm{n}^{\circ} 120$, p. 230-253.

Fuwa N., Edmonds C., Banik P. (2005). How inefficient are small-scale rice farmers in Eastern India really? Examining the effects of microtopography on technical efficiency estimates. East West Center, Working Papers, 30 p.

Gragasin M., Maruyama A., Marciano E., Fujiie M., Kikikuchi M. (2005). Irrigators'associations and farm productivity: a comparative study of two Philippine irri- 
gation systems. The Japanese Journal of Rural Economics, vol. 7, p. 1-17.

Kodde D.-A, Palm F.-C. (1986). Wald criteria for jointly testing equality and inequality restrictions. Chicago, the University of Chicago, Econometric Society, Econometrica, vol. 54, $\mathrm{n}^{\circ} 5$, p. $1243-1248$.

Kompas T. (2004). Market reform, productivity and efficiency in Vietnamese rice production. The Australian National University, Asia Pacific School of Economics and Government, $36 \mathrm{p}$.

Koopmans T.-C. (1951). An analysis of production as an efficient combination of activities. In T.-C. Koopmans (Éd.), «Activity analysis of production and allocution, Cowles commission for research in Economics », Wiley, New York, Monographe $\mathrm{n}^{\circ} 13$, p. 33-97.

Leibenstein H. (1966). Allocative efficiency versus X-Efficiency. Nashville, American Economic Association, The American Economic Review, vol. 56, p. 392-415. Meeusen W.-W., Van Den Broeck J. (1977). Efficiency estimation from Cobb-Douglas production functions with composed errors. Economics department of the University of Pennsylvania and Osaka
University institute of social and economic research association, International Economic Review, vol. 18, p. 435-444.

Morishima M. (1967). A few suggestions on the theory of elasticity. Nihon Hyronsha, Tokyo, Keizai Hyoron, ${ }^{\circ} 16$, p. 144-150.

Murillo-Zamorano L. (2004). Economic efficiency and frontier techniques. Blackwell, Oxford, Journal of Economic Surveys, vol. 18, $\mathrm{n}^{\circ} 10$, p. 33-77.

Ogundele O.-O., Okoruwa V.-O. (2004). Technical efficiency differentials in rice production technologies in Nigeria. Nigeria, University of Ibadan, 17 p.

Simon H.-A. (1947). A behavorial model of rational choice. Harvard University, Quarterly Journal of Economics, $\mathrm{n}^{\circ} 69$, p. 99-118.

Villano R., Fleming E. (2004). Analysis of technical efficiency in a rainfed lowland rice environnement in central Luzon Philippines using a stochastic frontier production function with a heteroskedastic error structure. University of New England, Working Paper Series in Agricultural and Ressource Economics, 28 p. 


\section{ANNEXES}

Annexe 1. Résultats des études sur l'efficience technique dans la culture rizicole

\begin{tabular}{|c|c|c|c|c|}
\hline Auteurs & Pays étudiés & Année & Variables étudiées & Résultats obtenus \\
\hline Audibert & Mali & $1997 b$ & $\begin{array}{l}\quad \text { PRODUCTION } \\
\text { Surface cultivée (en ha) } \\
\text { Main-d'œuvre familiale } \\
\text { Main-d'œuvre extra-familiale } \\
\text { Intrants (en valeur) } \\
\quad \text { EFFICIENCE TECHNIQUE } \\
\text { Age } \\
\text { Activités vivrières traditionnelles } \\
\text { Aménagement moderne des } \\
\text { parcelles } \\
\text { Taille du ménage } \\
\text { Bonne cohésion sociale } \\
\text { Santé (mauvaise) }\end{array}$ & $\begin{array}{l}\text { Effet significatif et positif } \\
\text { Effet significatif et positif } \\
\text { Effet significatif et positif } \\
\text { Effet significatif et positif } \\
\text { Effet non significatif } \\
\text { Effet significatif et négatif } \\
\text { Effet significatif et positif } \\
\text { Effet significatif et positif } \\
\text { Effet significatif et positif } \\
\text { Effet significatif et négatif }\end{array}$ \\
\hline $\begin{array}{l}\text { Gragasin } \\
\text { Maruyama } \\
\text { Marciano } \\
\text { Fujiie } \\
\text { et Kikikuchi }\end{array}$ & Philippines & 2005 & $\begin{array}{l}\quad \text { PRODUCTION } \\
\text { Surface cultivée (en ha) } \\
\text { Main-d'œuvre totale } \\
\text { Montant du capital } \\
\quad \text { EFFICIENCE TECHNIQUE } \\
\text { Localisation géographique } \\
\text { Age } \\
\text { Part du revenu issu des } \\
\text { activités rizicoles }\end{array}$ & $\begin{array}{l}\text { Effet significatif et positif } \\
\text { Effet significatif et positif } \\
\text { Effet significatif et positif } \\
\text { Effet significatif et positif } \\
\text { Effet significatif et positif } \\
\text { Effet significatif et négatif }\end{array}$ \\
\hline Kompas & Vietnam & 2004 & $\begin{array}{l}\quad \text { PRODUCTION } \\
\text { Stock de capital } \\
\text { Travail } \\
\text { Surface cultivée (en ha) } \\
\quad \text { EFFICIENCE TECHNIQUE } \\
\text { Taille de l'exploitation } \\
\text { Conditions du sol } \\
\text { Nombre de tracteurs }\end{array}$ & $\begin{array}{l}\text { Effet significatif et positif } \\
\text { Effet significatif et positif } \\
\text { Effet significatif et positif } \\
\text { Effet significatif et positif } \\
\text { Effet significatif et positif } \\
\text { Effet significatif et négatif }\end{array}$ \\
\hline $\begin{array}{l}\text { Villano et } \\
\text { Fleming }\end{array}$ & Philippines & 2004 & $\begin{array}{l}\quad \text { PRODUCTION } \\
\text { Surface cultivée (en ha) } \\
\text { Utilisation d'engrais } \\
\text { Travail } \\
\quad \text { EFFICIENCE TECHNIQUE } \\
\text { Age } \\
\text { Éducation } \\
\text { Revenus issus d'activités } \\
\text { non agricoles }\end{array}$ & $\begin{array}{l}\text { Effet significatif et positif } \\
\text { Effet significatif et positif } \\
\text { Effet significatif et positif } \\
\text { Effet non significatif } \\
\text { Effet non significatif } \\
\text { Effet significatif et négatif }\end{array}$ \\
\hline $\begin{array}{l}\text { Ogundele et } \\
\text { Okoruwa }\end{array}$ & Nigeria & 2004 & $\begin{array}{l}\quad \text { PRODUCTION } \\
\text { Surface cultivée (en ha) } \\
\text { Main-d'œuvre familiale } \\
\text { Main-d'œuvre saisonnière } \\
\text { Quantité d'engrais (en kg) } \\
\text { Age des agriculteurs } \\
\text { EFFICIENCE TECHNIQUE } \\
\text { Éducation } \\
\text { Années d'expérience } \\
\text { Taille du ménage }\end{array}$ & $\begin{array}{l}\text { Effet significatif et positif } \\
\text { Effet non significatif } \\
\text { Effet significatif et positif } \\
\text { Effet significatif et positif } \\
\text { Effet non significatif } \\
\text { Effet significatif et positif } \\
\text { Effet significatif et négatif } \\
\text { Effet non significatif }\end{array}$ \\
\hline
\end{tabular}


Annexe 2. Description et statistiques descriptives des variables retenues

\begin{tabular}{|c|c|}
\hline Dénomination & Définition $Y$ \\
\hline $\mathrm{Y}$ & Production totale de riz obtenue en kilogrammes sur l'ensemble de l'exploitation \\
\hline Xi & Facteurs de production \\
\hline $\mathrm{K}$ & Capital rudimentaire détenu au sein de l'exploitation (de 0 à 7) \\
\hline L & Main-d'œuvre familiale et employés permanents (nombre de personnes) \\
\hline MoT & Main-d'œuvre temporaire (nombre de jours de travailleurs temporaires employés) \\
\hline SuperRiz & Superficie de riz cultivé en hectares \\
\hline CapMod & Utilisation de capital moderne $=0$ si NON $;=1$ si OUI \\
\hline Ui & Variables retenues pour l'efficience technique \\
\hline REGHYD & $\begin{aligned} \text { Régime hydrique } & =0 \text { si régime pluvial } \\
& =1 \text { si régime irrigué }\end{aligned}$ \\
\hline RÉGION & $\begin{array}{l}\text { Exploitation dans une région naturelle favorable } \\
=1 \text { si OUI (Guinée maritime et Guinée forestière) } \\
=0 \text { si NON (Haute et Moyenne Guinée) }\end{array}$ \\
\hline INTRANTS & Utilisation d'au moins un intrant chimique $=0$ si NON $;=1$ si OUI \\
\hline SEXE & $\begin{aligned} \text { Sexe du chef de famille } & =0 \text { si chef de ménage féminin } \\
& =1 \text { si chef de ménage masculin }\end{aligned}$ \\
\hline AGE & Age du chef de ménage \\
\hline ALPHA & $\begin{aligned} \text { Niveau d'alphabétisation du chef de ménage } & =0 \text { ne sait pas lire ou écrire } \\
& =1 \text { sait lire ou écrire }\end{aligned}$ \\
\hline MonoC & Monoculture = 0 si NON ; = 1 si OUI \\
\hline ACTIFNONAGR & $\begin{array}{l}\text { Au moins un actif de l'exploitation pratique des activités non agricoles } \\
=0 \text { si NON ; = } 1 \text { si OUl }\end{array}$ \\
\hline CRÉDIT & $\begin{array}{l}\text { Crédit accordé par les institutions officielles ou non officielles } \\
=0 \text { si NON ; }=1 \text { si OUI }\end{array}$ \\
\hline NbreCHAMPS & Nombre total de champs constituant l'exploitation \\
\hline PROPRIÉTÉ & Propriété d'une parcelle = 0 si NON ; = 1 si OUI \\
\hline
\end{tabular}

\begin{tabular}{l|c|c|c|c|r}
\hline Variables & Observations & Moyenne & Ecart-type & Minimum & Maximum \\
\hline Production de riz (Y) & 828 & 2610,22 & 3143,66 & 80 & 51835,56 \\
\hline Capital rudimentaire & 828 & 3,83 & 0,998 & 1 & 7 \\
Travail permanent & 828 & 4,18 & 2,564 & 1 & 21 \\
Superficie de riz & 828 & 1,34 & 1,21 & 0,05 & 10,43 \\
Travail temporaire & 828 & 195,76 & 363,13 & 1 & 5820 \\
Capital moderne & 828 & 0,15 & 0,361 & 0 & 1 \\
\hline Régime hydrique & 828 & 0,14 & 0,347 & 0 & 1 \\
Région naturelle & 828 & 0,69 & 0,46 & 0 & 1 \\
Intrants chimiques & 828 & 0,07 & 0,247 & 0 & 1 \\
Sexe & 828 & 0,96 & 0,19 & 0 & 1 \\
Age & 828 & 47,18 & 14,56 & 18 & 92 \\
Alphabétisation & 828 & 0,23 & 0,422 & 0 & 1 \\
Monoculture & 828 & 0,47 & 0,5 & 0 & 1 \\
Actif non agricole & 828 & 0,40 & 0,490 & 0 & 1 \\
Crédit & 828 & 0,26 & 0,438 & 0 & 1 \\
Nombre de champs & 828 & 1,90 & 0,938 & 1 & 7 \\
Propriétaire & 828 & 0,86 & 0,199 & 0 & 1 \\
\hline
\end{tabular}

Source : conception de l'auteur, d'après les statistiques descriptives du RNA de la FAO réalisé en 2000-2001 
RECHERCHES

Charlotte FONTAN

Annexe 3. Résultats de l'analyse en frontière de production stochastique dans le cas d'une fonction Cobb-Douglas

\begin{tabular}{lccc}
\hline Variables $^{1}$ & & Coefficients $^{2}$ & $t$ de Student \\
\hline Coefficients agissant sur la fonction de production & $\beta_{0}$ & $8,09^{* * *}$ & 25,62 \\
Constante & $\beta_{1}$ & $0,12^{* * *}$ & 2,59 \\
Capital rudimentaire détenu (K) & $\beta_{2}$ & $-0,03$ & $-0,96$ \\
Nombre d'actifs agricoles permanents (L) & $\beta_{3}$ & $0,05^{* * *}$ & 4,84 \\
Nombre de jours d'actifs temporaires utilisés (MoT) & $\beta_{4}$ & $0,94^{* * *}$ & 34,19 \\
Superficie de riz cultivée (en Ha) (SuperRiz) & $\beta_{5}$ & $-0,08^{*}$ & $-1,84$ \\
Emploi de capital moderne (CapMOD) & & & \\
\hline Coefficients agissant sur l'inefficience technique 3 & $\delta_{0}$ & $1,11^{* * *}$ & 3,52 \\
Constante & $\delta_{1}$ & $-0,16^{* * *}$ & $-3,45$ \\
Régime hydrique & $\delta_{2}$ & 0,08 & 1,02 \\
Région naturelle favorable & $\delta_{3}$ & 0,02 & 0,37 \\
Utilisation d'au moins un intrant chimique & $\delta_{4}$ & 0,02 & 0,29 \\
Chef de ménage masculin & $\delta_{5}$ & $-0,003^{* * *}$ & $-2,59$ \\
Age du chef de ménage & $\delta_{6}$ & $-0,002$ & $-0,09$ \\
Etat d'alphabétisation & $\delta_{7}$ & $-0,09 * * *$ & $-2,76$ \\
Monoculture pratiquée & $\delta_{8}$ & 0,02 & 1,61 \\
Actif de l'exploitation dans activités non agricoles & $\delta_{9}$ & $-0,05^{*}$ & $-1,64$ \\
Recours à un crédit & $\delta_{10}$ & $-0,04^{* *}$ & $-1,98$ \\
Nombre total de champs & $\delta_{11}$ & $-0,15^{* *}$ & $-2,15$ \\
Propriété de champs & $\gamma$ & $0,54^{* * *}$ & 3,27 \\
\hline Gamma & &
\end{tabular}

Notes : (1) Variable exogène : la quantité de riz cultivée ; (2) Coefficient * Significatif à $10 \%$; **Significatif à $5 \%$; *** Significatif à $1 \%$; (3) Coefficient sur l'inefficience technique. On mesure bien ici la relation entre les différentes variables retenues et l'inefficience technique. Un signe négatif expliquera donc un effet défavorable de la variable sur I'inefficience technique et donc finalement un effet positif pour l'efficience; (4) Gamma représente la part de la variance de $\mathrm{U}$, qui désigne les facteurs de l'inefficience technique, dans la variance totale. Ce coefficient permet de discuter de la cohérence statistique du modèle.

Source : conception de l'auteur, d'après les calculs effectués sur FRONTIER 4.1 à partir du RNA de la FAO réalisé en 2000-2001 